\title{
A novel variant of VEGFR2 identified by a pan-cancer screening of recurrent somatic mutations in the catalytic domain of tyrosine kinase receptors enhances tumor growth and metastasis
}

\author{
Elisabetta Grillo ${ }^{\text {a,1, }{ }^{* *}}$, Michela Corsini ${ }^{\text {a, }}{ }^{\text {, Cosetta Ravelli }}{ }^{\text {a,b }}$, Margherita di Somma ${ }^{\text {a }}$ \\ Luca Zammataro $^{c}$, Eugenio Monti ${ }^{\mathrm{a}}$, Marco Presta ${ }^{\mathrm{a}}$, Stefania Mitola ${ }^{\mathrm{a}, \mathrm{b}, *}$

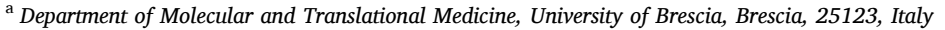 \\ ${ }^{\mathrm{b}}$ Laboratory for Preventive and Personalized Medicine (MPP Lab), University of Brescia, 25123, Italy \\ ${ }^{\mathrm{c}}$ Department of Obstetrics, Gynecology and Reproductive Sciences, Yale School of Medicine, New Haven, CT 06510, USA
}

\section{A R T I C L E I N F O}

\section{Keywords:}

Cancer/mutation/tyrosine kinase receptor/ VEGFR2

\begin{abstract}
A B S T R A C T
In cancer genomics, recurrence of mutations in gene families that share homologous domains has recently emerged as a reliable indicator of functional impact and can be exploited to reveal the pro-oncogenic effect of previously uncharacterized variants. Pan-cancer analyses of mutation hotspots in the catalytic domain of a subset of tyrosine kinase receptors revealed that two infrequent mutations of VEGFR2 (R1051Q and D1052N) recur in analogous proteins and correlate with reduced patient survival. Functional validation showed that both R1051Q and D1052N mutations increase the enzymatic activity of VEGFR2. The expression of VEGFR2 ${ }^{\mathrm{R} 1051 \mathrm{Q}}$ potentiates the PI3K/Akt signaling axis in cancer cells, increasing their tumorigenic potential in vitro and in vivo. In addition, it confers to cancer cells an increased sensitivity to the VEGFR2-targeted tyrosine kinase inhibitor Linifanib. In the context of an efficacious application of anti-cancer targeted therapies, these findings indicate that the screening for uncharacterized mutations, like VEGFR2 ${ }^{\mathrm{R} 1051 \mathrm{Q}}$, may help to predict patient prognosis and drug response, with significant clinical implications.
\end{abstract}

\section{Introduction}

One powerful approach for a systematic identification of clinically relevant somatic gene variants in cancer is based on the mutational analysis of protein domains, evolutionary conserved units of proteins related in terms of sequence, structure and function [1,2]. The similarity of protein domains set the bases for the creation of various databases, including Pfam (https://pfam.xfam.org/) [3], that classify proteins into families and allow to transfer the functional information from an experimentally characterized sequence to uncharacterized ones. For example, hotspots of mutations recurring in the same position across homologous domains can be exploited to pinpoint novel uncharacterized variants that are equivalent to well-known mutations in other proteins of the same family [4-6]. This approach has allowed the detection and characterization of previously unknown oncogenic and/or actionable cancer variants of tyrosine kinase receptors (RTKs), including
TGFBR, HER2 and VEGFR2 [7,8], a protein family commonly dysregulated in tumors and an important target for cancer therapy $[9,10]$. Remarkably, case reports and NGS analyses have previously described various mutations of VEGFR2, including L840F, R961W, R1032Q and S1100F, some of which correlate with tumor growth and altered response to tyrosine kinase inhibitors (TKi) [7,11,12].

Here, we used the recently developed Low-frequency Mutation Analysis via Consensus Alignment (LowMACA) bioinformatics tool [6] to analyze recurrent mutations in the tyrosine kinase domain (TKD) of a subset of RTKs. This allowed the identification of two mutation hotspots located in the activation loop of the TKD, corresponding to the uncharacterized R1051Q and D1052N mutations of VEGFR2, a key player in angiogenesis and cancer [13-15]. In our study, functional characterization showed that both R1051Q and D1052N mutations of VEGFR2 entail a "gain-of-function" by increasing the activity of the receptor. Moreover, when expressed in cancer cells, VEGFR2 ${ }^{\mathrm{R} 1051 \mathrm{Q}}$

\footnotetext{
* Corresponding author. Department of Molecular and Translational Medicine, University of Brescia, Brescia, 25123, Italy.

** Corresponding author. Department of Molecular and Translational Medicine, University of Brescia, Brescia, 25123, Italy.

E-mail addresses: elisabetta.grillo@unibs.it (E. Grillo), stefania.mitola@unibs.it (S. Mitola).

1 Equally contributed.
} 
promotes their pro-oncogenic capacity and augments the tumor sensitivity to a VEGFR2-targeted TKi. Our observations confirm that mutation recurrence across analogous proteins is predictive of functional impact.

\section{Material and methods}

Bioinformatics analyses. MutationAligner (http://mutationaligner. org/) [4] and Low-frequency Mutations Analysis via Consensus Alignment (LowMACA) (http://www.bioconductor.org/packages/release/ bioc/html/LowMACA.html) [6] bioinformatics tools were used to identify the amino acids recurrently mutated in the PK_Tyr_Ser-Thr domain obtained from Pfam database (Pfam PF07714). After the generation of a multiple sequence alignment, both tools summed all mutations identified in cancer samples on properly aligned amino acids. Built-in statistical models were used to assess the statistical significance of mutation hotspots [described in Refs. [4-6], http://mutation aligner.org/methods].

MutationAligner enables to explore somatic mutations identified from TCGA variant data processed by cBioPortal spring 2015 and all the proteins belonging to a given Pfam must be analyzed together. At variance, LowMACA retrieves mutation data from TCGA, COSMIC and cBioPortal or custom databases and it allows to select a customized group of proteins of interest within a given protein family to run the analysis.

The overall survival of patients with tumor harboring or not the mutations identified by LowMACA was retrieved from cBioPortal.

Mutagenesis. pBE_hVEGFR2 plasmids encoding wild-type hVEGFR2 [NM_002253.2] and hVEGFR2-YFP, were provided by Prof. Kurt Ballmer-Hofer (Paul Scherrer Institut, Villigen, Switzerland) and by Dr. Kalina Hristova (Johns Hopkins University, Baltimore, USA), respectively. R1051Q and D1052N point mutated plasmids were generated using primers listed in Table 1 by QuikChange Lightning Site-directed Mutagenesis Kit (Agilent Technologies).

Cell cultures. Chinese hamster ovary (CHO) cells were grown in Ham's F-12 supplemented with 10\% fetal calf serum (FCS) and penicillin/streptomycin. Fetal bovine aortic endothelial GM7373 cells, porcine aortic endothelial cells (PAE) and human breast adenocarcinoma cell line MCF7 (purchased from ATCC) were grown in DMEM supplemented with 10\% FCS (Invitrogen) and penicillin/streptomycin. Human melanoma Sk-Mel-31 cells (characterized for the expression of B-Raf, N-Ras and VEGFR2 wild type) were provided by Memorial Sloan Kettering Cancer Center and were grown in RPMI supplemented with $10 \%$ FCS, non-essential amino-acids and penicillin/streptomycin.

Cells were stably transfected with pBE_hVEGFR2, pBE_hVEGFR2 ${ }^{\text {R1051Q }}$ or pBE_hVEGFR2 ${ }^{\text {D1052N }}$ plasmid using FuGENE (Promega). Transfected cell lines were maintained in $0.5 \mathrm{mg} / \mathrm{ml}$ geneticin. Different transfections achieved similar levels of VEGFR2 expression.

VEGFR2 kinase assay. VEGFR2-immunoprecipitated fractions of CHO cells transiently expressing VEGFR2 variants were obtained using limiting amounts of anti-VEGFR2 antibody (sc-6251, Santa Cruz
Biotechnology, Inc) and assayed using ADP-Glo Kinase Assay + KDR Kinase Enzyme System according to manufacturer's instructions (Promega). Bioluminescent signal was measured with EnSight Multimode Plate Reader (PerkinElmer).

Immunoprecipitation and Western blot analyses. Immunoprecipitated fractions (starting from $1 \mathrm{mg}$ of total proteins) or total lysates were separated by SDS-PAGE and probed with specific antibodies (Table 2). Chemiluminescent signal was acquired by ChemiDoc ${ }^{\mathrm{TM}}$ Imaging System (BioRad).

Fluorescence Recovery After Photobleaching (FRAP). CHO cells transiently expressing VEGFR2 variants were seeded at $5.0 \times 10^{5}$ cells/ $\mathrm{mL}$ in $\mu$-slides (ibidi) and analyzed using LSM880 confocal microscope equipped with an incubation chamber (Carl Zeiss). Images were recorded with $2 \%$ of the intensity of the $514-n m$ line. YFP was bleached using 50 -iteration at $100 \%$ intensity of the 514 -nm line. Fluorescence recovery in bleached areas was followed for 8 minutes (1 image/minute) and analyzed using FRAP tool of Zen black software (Carl Zeiss) [16].

Cytofluorimetric analyses. Membrane expression of VEGFR2 was assessed by staining with anti-VEGFR2 antibody (\#ab11939, Abcam) and analyzed using the MACSQuantAnalyzer (Miltenyi Biotec). Propidium iodide positive cells were excluded. Data were analyzed by FlowJo software.

Cell proliferation and clonogenic assays. $6 \times 10^{3} \mathrm{cells} / \mathrm{cm}^{2}$ were cultured in growth medium in the absence or the presence of indicated inhibitors for 6 days. Cell number was evaluated by cell counting or by crystal violet colorimetric assay (OD at $595 \mathrm{~nm}$ ). For 2D clonogenic assays, $1 \times 10^{2}$ cells $/ \mathrm{cm}^{2}$ were cultured in growth medium. Samples were stained with $0.1 \%$ crystal violet and analyzed by ImageJ software. For 3D clonogenic assays, cells were embedded at $50 \mathrm{cells} / \mathrm{cm}^{3}$ in Cultrex matrix or soft agar gel. The percentage of colony-forming cells or spheroid average size were calculated by ImageJ analysis software after 7 or 15 days respectively.

Table 2

List of antibodies used in this study.

\begin{tabular}{|c|c|c|}
\hline Antibody & Catalog number & Supplier \\
\hline Anti-total-phospho-tyrosine & \#05-321 & EMD Millipore Corp \\
\hline Anti-VEGFR2 & \#9698S & Cell signaling Technology \\
\hline Anti-FAK & \#AHO0502 & ThermoFisher Scientific \\
\hline Anti-phospho-Akt ${ }^{\text {Ser473 }}$ & \#4060 & Cell signaling Technology \\
\hline $\begin{array}{l}\text { Anti-phospho-p70 S6 } \\
\text { Kinase }^{\text {Thr421/Ser424 }}\end{array}$ & \#9204 & Cell signaling Technology \\
\hline Anti-phospho- $\beta$-Catenin ${ }^{\text {Ser552 }}$ & \#9566 & Cell signaling Technology \\
\hline Anti-phospho-CREB ${ }^{\text {Ser133 }}$ & \#9198 & Cell signaling Technology \\
\hline Anti- $\beta$-Catenin & \#9562 & Cell signaling Technology \\
\hline Anti-Mcl-1 & \#AB2910 & Chemicon International \\
\hline Anti-GAPDH & \#sc-25778 & Santa Cruz Biotechnology \\
\hline Anti-CD31 & \#DIA-310 & DIANOVA GmbH \\
\hline Anti- $\alpha$-SMA-FITC & \#F3777 & Sigma \\
\hline Anti-phospho-VEGFR2 $2^{\text {Tyr951 }}$ & \#sc-16628-R & Santa Cruz Biotechnology \\
\hline
\end{tabular}

Table 1

List of primers used in this study.

\begin{tabular}{|c|c|c|}
\hline Gene & Forward $\left(5^{\prime}-3^{\prime}\right) \mathrm{s}$ & Reverse $\left(5^{\prime}-3^{\prime}\right)$ \\
\hline Human_Collagen1A1 & AAGAGGAAGGCCAAGTCGAG & AGATCACGTCATCGCACAAC \\
\hline Human_Collagen1A2 & TTTAATTTTTCTGCTTGCCCA & CAAAACACTTTCCCATGAGTG \\
\hline Human_Laminin & GGCCCTGTGTTTGTAAGGAA & TCTTGCTGAGACGGGATCTT \\
\hline Human_Fibronectin & TATGTGGTCGGAGAAACGTG & TCCTTGTGTCCTGATCGTTG \\
\hline human_extra domain-B containing fibronectin (EDB) & CCACCATTATTGGGTACCGC & CGCATGGTGTCTGGACCAATG \\
\hline human_MMP2 & GTATGGCTTCTGCCCTGAGA & CACACCACATCTTTCCGTCA \\
\hline murine_Collagen $1 \mathrm{~A} 1$ & GGATCGACCCTAACCAAGGC & CGTACTCGAACGGGAATCCAT \\
\hline murine_Collagen $1 \mathrm{~A} 2$ & GGTGAGCCTGGTCAAACGG & ACTGTGTCCTTTCACGCCTTT \\
\hline murine_Fibronectin & ATACCGTTGTCCCAGAGGTG & GGAAGAGTTTAGCGGGGTCC \\
\hline human_GAPDH & GAAGGTCGGAGTCAACGGATT & TGACGGTGCCATGGAATTTG \\
\hline Mutagenesis_R1051Q & GTGACTTTGGCTTGGCCCAGGATATTTATAAAGATCCA & TGGATCTTTATAAATATCCTGGGCCAAGCCAAAGTCCAC \\
\hline Mutagenesis_D1052N & TCTGGATCTTTATAAATATTCCGGGCCAAGCCAAGTC & GACTTTGGCTTGGCCCGGAATATTTATAAAGATCCAGA \\
\hline
\end{tabular}


In vivo tumorigenesis. In vivo experiments were approved by the Italian "Ministero della Salute" and performed in accordance with the OPBA (Organismo Preposto al Benessere Animale) guidelines of the University of Brescia. $4 \times 10^{6}$ Sk-Mel-31-VEGFR2 ${ }^{\mathrm{WT}}$ or Sk-Mel-31VEGFR2 ${ }^{\mathrm{R} 1051 \mathrm{Q}}$ cells were injected subcutaneously (s.c.) into the dorsolateral flank of NOD/SCID mice (Envigo). When indicated, animals received daily oral gavage with $100 \mu \mathrm{L}$ of vehicle (corn oil) or $5 \mathrm{mg} / \mathrm{kg}$ of Linifanib (Selleckchem). Tumor volume was measured with calipers and calculated according to formula $\mathrm{V}=\left(\mathrm{Dxd}^{2}\right) / 2$, where $\mathrm{D}$ and $\mathrm{d}$ are the major and minor perpendicular tumor diameters, respectively. At the end of the experimental procedure, tumors were harvested, weighed, and processed for further analyses.

Experimental metastasis. Six- to eight-week old NOD/SCID mice (Envigo) were injected intravenously (i.v.) with parental or F1 generation Sk-Mel-31-VEGFR2 ${ }^{\text {WT }}$ or Sk-Mel-31-VEGFR2 ${ }^{\text {R1051Q }}$ cells $\left(3 \times 10^{5}\right.$ cells/mouse) in $100 \mu \mathrm{L}$ of PBS. After 10 weeks, mice were sacrificed, lungs were harvested and superficial metastatic nodules were counted.

Histological analyses. Formalin-fixed paraffin-embedded tissue sections were stained with hematoxylin and eosin (H\&E) or Masson's trichrome or antibodies listed in Table 2. Nuclei were counterstained with 4',6-diamidino-2-phenylindole (DAPI, Sigma). Images were captured using a Axiovert200 M microscope (Carl Zeiss) and analyzed by ImageJ software. For Second Harmonic Generation Imaging, images were captured using LSM880 two-photon microscope equipped with a Plan-Neofluar 20X/0.5 NA objective (Carl Zeiss).

Antibody arrays. Total lysates $(500 \mu \mathrm{g})$ from Sk-Mel-31-VEGFR2 ${ }^{\mathrm{WT}}$ or Sk-Mel-31-VEGFR2 ${ }^{\mathrm{R} 1051 \mathrm{Q}}$ cell-derived tumors were incubated with the human phospho-kinase array kit or with the human angiogenesis array kit (R\&D Systems) according to manufacturer's instructions. Chemiluminescent signal was analyzed using the ImageJ software.

$\boldsymbol{R T - q P C R}$. Total RNA was extracted using TRIzol Reagent (Invitrogen) according to manufacturer's instructions. Two $\mu \mathrm{g}$ of total RNA were retro-transcribed with MMLV reverse transcriptase (Invitrogen) using random hexaprimers. Then, cDNA was analyzed by qPCR using the primers listed in Table $1.2^{-\Delta \Delta \mathrm{Ct}}$ was calculated using human_GAPDH as housekeeping. Data are expressed as relative expression ratios.

Scratch and invasion assays. Confluent monolayers were scratched with a $200 \mu \mathrm{L}$ tip and incubated in growth medium. After 16 or $24 \mathrm{~h}$, the newly covered area was measured by ImageJ software. For invasion assay, 1000-cell spheroids were prepared in $20 \%$ methylcellulose medium, embedded in fibrin gel and incubated for $24 \mathrm{~h}$ in growth medium. Invaded/scattered area was measured by ImageJ software.

Matrix metalloproteinase (MMP) activity assay. The activity of MMP2 was evaluated on cell supernatants by gel zymography as previously described in Ref. [17]. Densitometric analysis was performed with ImageJ software.

Statistical analyses. Student's $t$-test for unpaired data (2-tailed) was used to test the probability of significant differences between two groups of samples. Differences were considered significant when $\mathrm{p}<0.05$, unless otherwise specified.

\section{Results}

\subsection{Pan-cancer analysis of recurrent mutations in the catalytic domain of tyrosine kinase receptors detects two novel variants of VEGFR2}

To identify previously uncharacterized cancer-related RTK mutations, we looked at somatic mutations recurring in the TKD (PK_Tyr_SerThr domain obtained from Pfam database - Pfam entry: PF07714) of a subset of RTKs. To this aim, we exploited the bioinformatics tool LowMACA that combines the mutations of various proteins sharing the same functional domain to identify conserved residues that harbor clustered mutations in multiple sequence alignments [6]. As shown in Fig. 1A-C, LowMACA aligned the TKD sequence of VEGFR2, EGFR, PDGFRB, PDGFRA, FLT1,3,4, FGFR1,2,3,4, and TIE receptors and generated a consensus sequence. Next, it retrieved somatic missense mutations of the selected RTKs from TCGA, COSMIC and cBioPortal databases of human cancer samples, and summed them on the properly aligned amino acid sequence. A built-in statistical analysis allowed to identify ten significant mutation hotspots along the aligned domain (red asterisks, Fig. 1C, see also Data in Brief). Among them, the mutations at position 255 and 256, both present in FGFR1-4, FLT3, FLT4, PDGFRB, EGFR, TIE1 and VEGFR2 receptors, are located in the activation loop of the TKD and significantly reduce the overall survival when occurring in cancer patients (Fig. 1D-F). This supports the hypothesis that mutations at position 255 and 256 may result in constitutively active RTKs and may drive tumor progression. Accordingly, the analysis of the entire PF07714 protein family by the MutationAligner web-resource [4,5], an additional tool for the search of mutation hotspots in protein domains, converged to identify positions 255 and 256 as recurrently mutated in this protein family (see Data in Brief). Notably, these positions correspond to two amino acid residues of the B-Raf oncogene that undergo the well-characterized activating mutations T599R/I and V600E [18]. This prompted us to assess whether mutations at positions 255 and 256 could have a similar functional impact on other kinases. A scientific literature search indicated that the effect of these mutations on FGFRs, FLT3-4 and EGFR function had been already addressed [19-24] while their impact on VEGFR2 activity remains to be elucidated. Given the central role of VEGFR2 in angiogenesis and tumor growth, we investigated the biological effects of mutations of positions 255 and 256 in VEGFR2, which correspond to the previously uncharacterized R1051Q and D1052N variants of the receptor.

\subsection{R1051Q and D1052N of VEGFR2 are activating mutations}

To address the impact of R1051Q and D1052 N substitutions on VEGFR2 function, we tested the kinase activity of the mutated receptors in comparison with wild-type VEGFR2 (VEGFR2 ${ }^{\mathrm{WT}}$ ) when expressed in CHO cells. As anticipated, both VEGFR2 ${ }^{\text {R1051Q }}$ and VEGFR2 ${ }^{\text {D1052N }}$ mutants display a significantly higher basal enzymatic activity and increase the levels of protein tyrosine phosphorylation when compared to VEGFR2 ${ }^{\text {WT }}$ (Fig. 2A-B). In addition, fluorescence recovery after photobleaching (FRAP) analysis showed that both mutated YFPVEGFR2 ${ }^{\mathrm{R} 1051 \mathrm{Q}}$ and YFP-VEGFR2 ${ }^{\mathrm{D} 1052 \mathrm{~N}}$ receptors have reduced lateral mobility, similar to that observed for VEGF-stimulated YFP-VEGFR2 ${ }^{\text {WT }}$ (Fig. 2C) also shown in Ref. [16].

VEGFR2 is a major mediator of pro-angiogenic signaling in vascular endothelial cells (ECs) [13]. To validate R1051Q and D1052N as "gain-of-function" mutations, we expressed the VEGFR2 ${ }^{\mathrm{R} 1051 \mathrm{Q}}$ or VEGFR2 ${ }^{\text {D1052N }}$ mutants in ECs from different sources and assessed VEGFR2-associated cell behaviors. Again, both VEGFR2 mutants increase the levels of protein tyrosine phosphorylation. Furthermore, ECs expressing VEGFR2 mutants are endowed with augmented proliferative and migratory capacities when compared to VEGFR2 ${ }^{\mathrm{WT}}$ transfectants (Fig. SI). Overall, these findings indicate that R1051Q and D1052N mutations are activating mutations that increase the activity of VEGFR2 even in the absence of exogenous stimulation. Given its more prominent impact on cell transfectants, we decided to focus our attention on the R1051Q mutation.

\subsection{Expression of VEGFR2 $2^{R 1051 Q}$ increases tumor cell proliferation and migration}

To investigate whether the VEGFR2 $2^{\mathrm{R} 1051 \mathrm{Q}}$ mutation might drive tumor cell proliferation, VEGFR2 ${ }^{\mathrm{WT}}$ or VEGFR2 ${ }^{\mathrm{R} 1051 \mathrm{Q}}$ receptors were expressed in B-Raf wild-type human breast adenocarcinoma MCF7 cells and melanoma Sk-Mel-31 cells. It must be pointed out that MCF7 cells do not express detectable levels of endogenous VEGFR2 (Fig. SIIA). At variance, Sk-Mel-31 cells express basal levels of the wild-type receptor (Fig. SIII), thus allowing to mimic the heterozygous status of the mutation found in cancer. VEGFR2 ${ }^{\mathrm{R} 1051 \mathrm{Q}}$ expression results in increased MCF7 and Sk-Mel-31 cell proliferation when compared to VEGFR2 ${ }^{\text {WT }}$ 
A

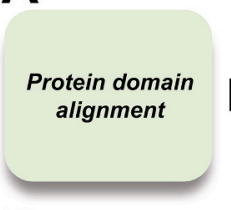

B

Pfam proteins:

EGFR

PDGFRB

FGFR1

PDGFRA

FLT1

FGFR2

FGFR4

FGFR3

TIE1

FLT4

VEGFR2

FLT3

Consensus
LowMACA workflow
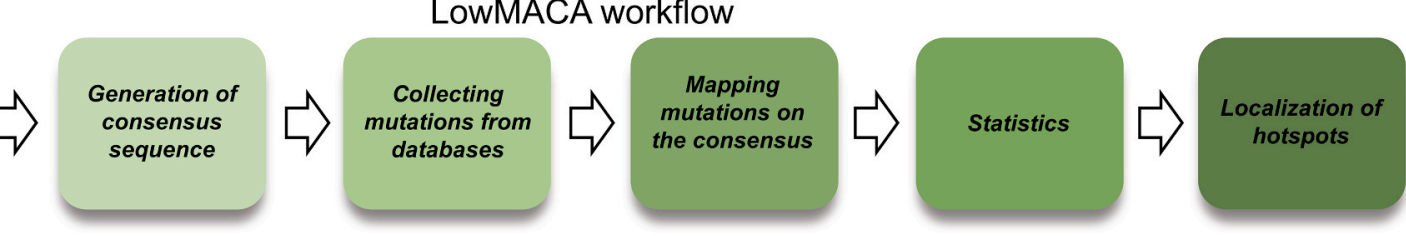

gap GAIVLMPFYWHCKRDEQNST

TKD (PK_Tyr_Ser-Thr domain, Pfam entry: PF07714)
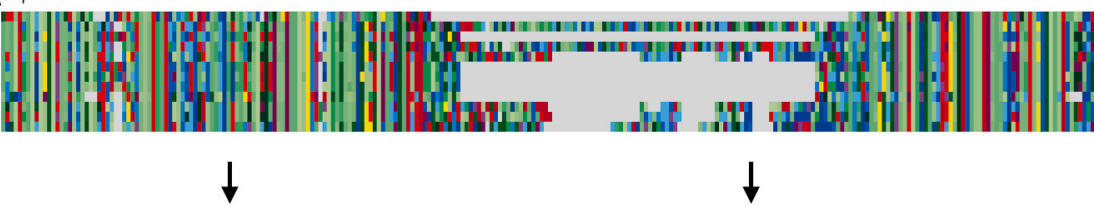

\section{$\downarrow$}

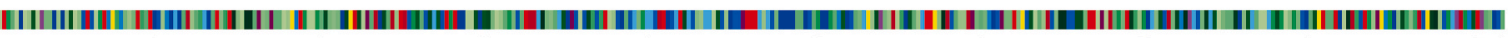

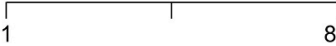

80

160

240

320

Position (aligned consensus sequence)

C

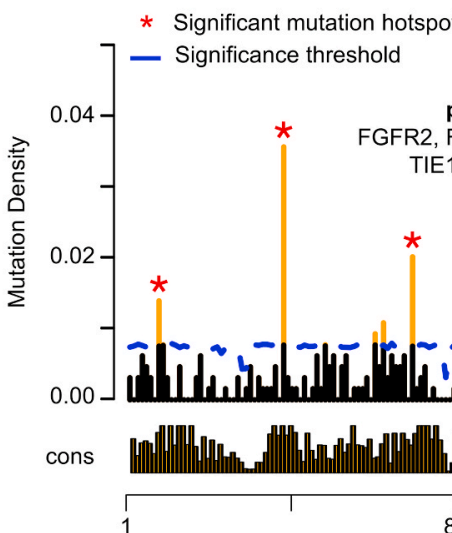

LowMACA Output: Global p-value: $4.2 \mathrm{e}-18$

pos: 253

EGFR, VEGFR2

pos: 255

FGFR1, FGFR4, FGFR3, FLT4, VEGFR2, PDGFRB, TIE1

FGFR4, FLT4, PDGFRA TIE1, VEGFR2 pos: 256

EGFR, FGFR1, FGFR2, FLT3, FLT4, VEGFR2

pos: 304

FGFR3, PDGFRA, VEGFR2

FLT1, PDGFRB
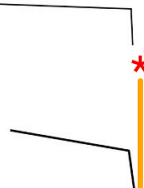

-

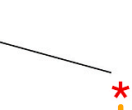

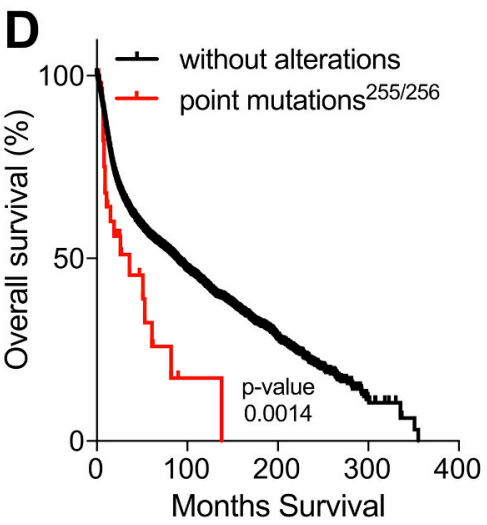

E \begin{tabular}{l|c|c} 
Position & $\mathbf{2 5 5}$ & $\mathbf{2 5 6}$ \\
\hline VEGFR2 & R1051Q & D1052N \\
\hline FGFR1 & R646L & D647N \\
\hline FGFR2 & - & D650H \\
\hline FGFR3 & R640W & - \\
\hline FGFR4 & R635S & - \\
\hline FLT3 & - & D835H/Y/E \\
\hline FLT4 & R1060Q & D1061N \\
\hline TIE1 & R1002W/Q & - \\
\hline PDGFRA & - & D842H \\
\hline PDGFRB & R849G & - \\
\hline EGFR & - & L861Q/R
\end{tabular}

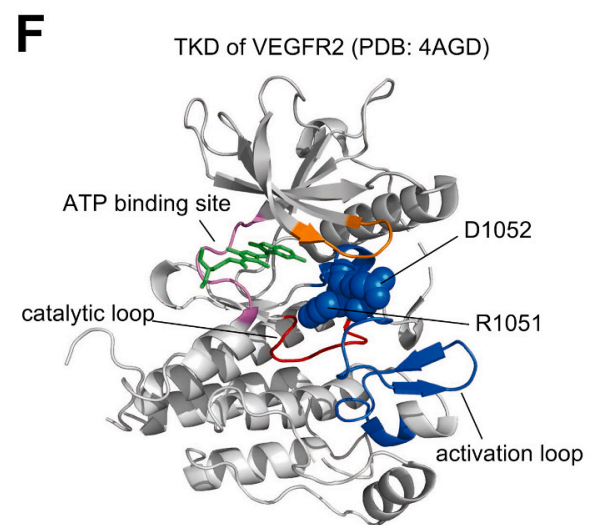

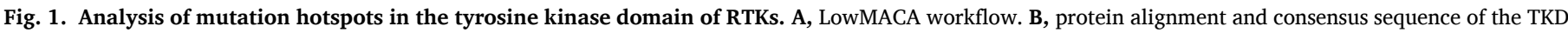

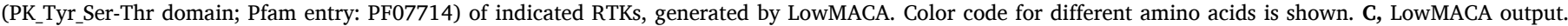

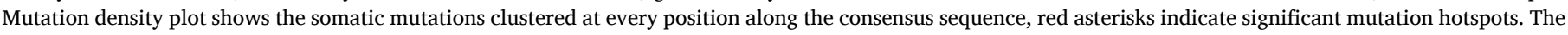

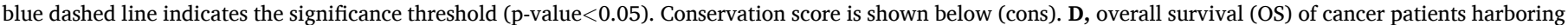

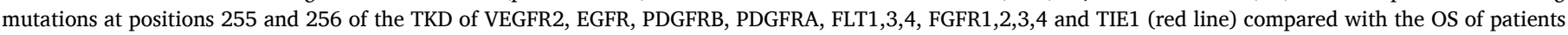

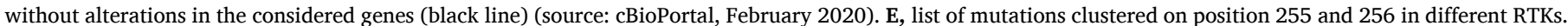

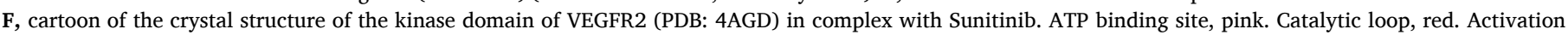

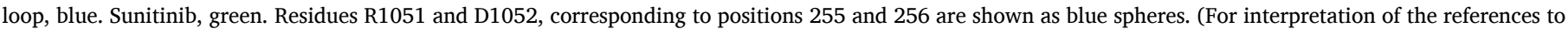
color in this figure legend, the reader is referred to the Web version of this article.) 

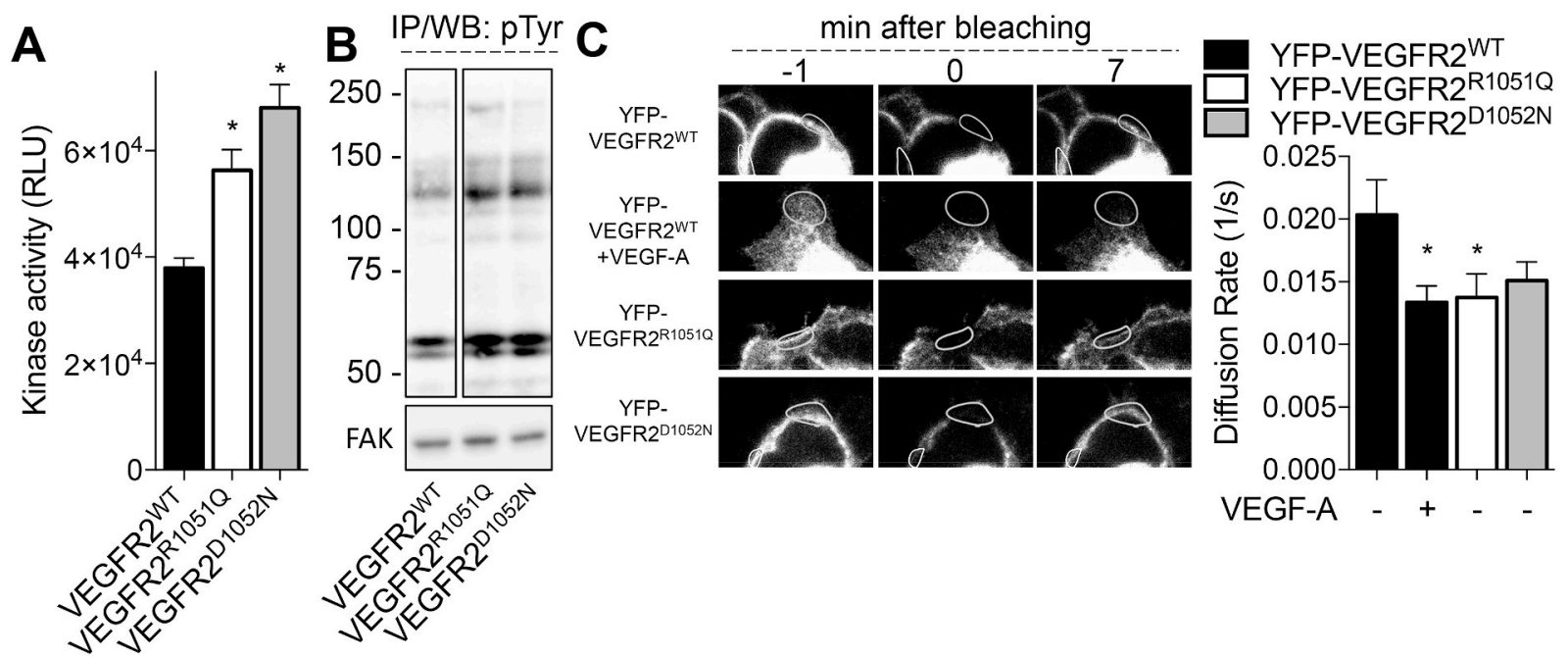

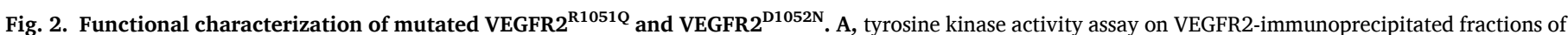

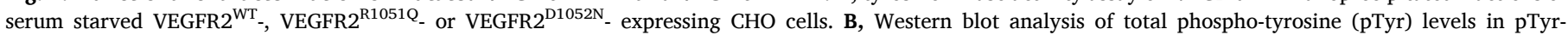

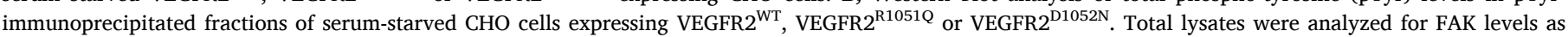

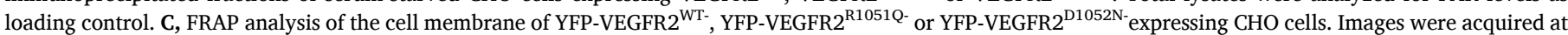

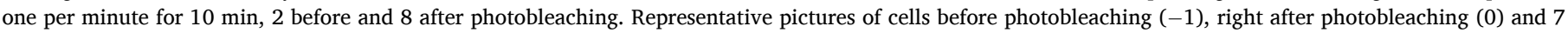

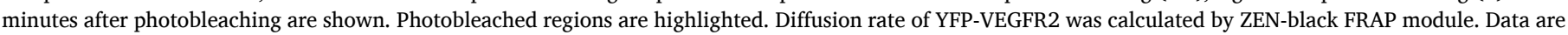
shown as mean \pm SEM. *, $\mathrm{p}<0.05$, Student's $t$-test versus VEGFR2 ${ }^{\mathrm{WT}}$.

transfectants (Fig. SIIB and Fig. 3A). Moreover, MCF7 ${ }^{\text {R1051Q }}$ and Sk-Mel31-VEGFR2 ${ }^{\mathrm{R} 1051 \mathrm{Q}}$ cells showed a greater clonogenic capacity both in anchorage-dependent and -independent assays (Fig. SIIC and Fig. 3B-C). In addition, Sk-Mel-31-VEGFR2 ${ }^{\text {R1051Q }}$ cells migrate faster than Sk-Mel31-VEGFR2 ${ }^{\text {WT }}$ cells (Fig. 3D) and show a more pronounced invasive capacity when embedded in a 3D fibrin gel (Fig. 3E). This increased invasive capacity is, at least in part, explained by the higher expression and activity of matrix metalloproteinase 2 (MMP2) (Fig. 3F-G), a collagenase highly associated with tumor dissemination and invasiveness $[25,26]$.

\subsection{Expression of VEGFR2 $2^{R 1051 Q}$ drives tumor growth and metastasis}

We next addressed the impact of the R1051Q mutation of VEGFR2 in an in vivo tumor model. As shown in Fig. 4A, Sk-Mel-31-VEGFR2 ${ }^{\mathrm{R} 1051 \mathrm{Q}}$ cells implanted subcutaneously (s.c.) in NOD/SCID mice grow faster than control cells. Immunofluorescence analysis of phospho-VEGFR2 in tumor sections confirmed that the mutated VEGFR2 is highly phosphorylated (Fig. 4B).

Sk-Mel-31-VEGFR2 ${ }^{\mathrm{WT}}$ and SK-Mel-31-VEGFR2 ${ }^{\mathrm{R} 1051 \mathrm{Q}}$-derived tumors exhibit a different tissue architecture (Fig. 4B). SK-Mel-31VEGFR2 ${ }^{\mathrm{R} 1051 \mathrm{Q}}$-derived tumors are highly vascularized with immature CD $31^{+}$blood vessels devoid of $\alpha$-SMA ${ }^{+}$mural cells (Fig. 4B). Accordingly, angiogenesis antibody array analysis showed that Sk-Mel-31VEGFR2 ${ }^{\text {R1051Q }}$ tumors express higher levels of various pro-angiogenic factors, including angiogenin, PDGF-A, and PlGF, whereas VEGF is slightly decreased (Fig. 4C). Also, collagen fibrils in the extracellular matrix (ECM) are more abundant and more organized in tumors derived from Sk-Mel-31-VEGFR2 ${ }^{\mathrm{R} 1051 \mathrm{Q}}$ cells, as demonstrated by Masson's trichrome staining and second harmonic generation imaging (SHG) (Fig. 4D-E). This was accompanied by a higher expression of both parenchymal and stromal COL1A1, COL1A2, laminin, fibronectin, and extra-domain-B (EDB) containing fibronectin isoform genes (Fig. 4F-G).

A phospho-kinase array on tumor xenografts demonstrated that the expression of VEGFR2 ${ }^{\mathrm{R} 1051 \mathrm{Q}}$ in Sk-Mel-31 cells induces the activation of a pro-oncogenic intracellular signaling that encompasses the activation of the PI3K/Akt/mTOR pathway, as shown by the increased phosphorylation of Akt, p70-S6-Kinase, WNK1, GSK- $\alpha / \beta$, $\beta$-catenin and CREB (Fig. 5A). To confirm the activation of the PI3K/Akt/mTOR pathway in tumor parenchyma, we performed Western blot analyses on Sk-Mel-31 transfectants. These analyses confirmed the activation of Akt and its targets and showed an increase in the levels of the anti-apoptotic protein Mcl-1 following VEGFR2 ${ }^{\text {R1051Q }}$ expression (Fig. 5B). Accordingly, the AKT/mTOR pathway inhibitors AZD8055, rapamycin, and everolimus inhibit the proliferation of Sk-Mel-31-VEGFR2 $2^{\text {R1051Q }}$ cells with higher efficacy when compared to Sk-Mel-31-VEGFR2 ${ }^{\text {WT }}$ cells, confirming the hyper-activation of the Akt/mTOR pathway in melanoma cells following VEGFR2 ${ }^{\text {R1051Q }}$ expression (Fig. 5C).

Finally, when Sk-Mel-31-VEGFR2 ${ }^{\mathrm{R} 1051 \mathrm{Q}}$ cells and their $\mathrm{F} 1$ generation were injected intravenously (i.v.) in NOD/SCID animals, they displayed a more potent lung metastatic capacity compared to VEGFR2 ${ }^{\mathrm{WT}}$ transfectants (Fig. 6).

\subsection{Expression of VEGFR2 $2^{R 1051 Q}$ increases the sensitivity of melanoma cells to VEGFR2-targeted TKi}

To assess whether the R1051Q mutation of VEGFR2 affects its response to TKi, Sk-Mel-31 cells expressing either the mutated or the wild-type receptor were treated with increasing doses of the ATPcompetitive VEGFR2 inhibitor Linifanib [27]. In vitro, Linifanib exerts a more pronounced inhibitory effect on the colony formation capacity of Sk-Mel-31-VEGFR2 ${ }^{\text {R1051Q }}$ cells than on cells expressing VEGFR2 ${ }^{\text {WT }}$ ( $\sim 80 \%$ versus $\sim 40 \%$ inhibition) (Fig. $7 \mathrm{~A}$ ). In vivo, Linifanib inhibits the growth of Sk-Mel-31-VEGFR2 ${ }^{\mathrm{R} 1051 \mathrm{Q}}$ tumor grafts being instead ineffective on the growth of tumors expressing the wild-type receptor (Fig. 7B). Altogether, these data indicate that the VEGFR2 ${ }^{\mathrm{R} 1051 \mathrm{Q}}$ mutation confers an increased sensitivity of melanoma cells to the VEGFR2-targeted TKi Linifanib.

\section{Discussion}

Protein domain hotspot analysis allows to detect unknown mutations in one protein that are equivalent to well-known variants of other proteins, to identify new mutation hotspots and to predict the functional impact of mutations on target proteins, thus making possible to link specific mutations to a particular cancer phenotype and/or drug response. A pan-cancer bioinformatics analysis of recurrent mutations in the TKD of a subset of RTKs led us to discover 10 significant hotspots 
A
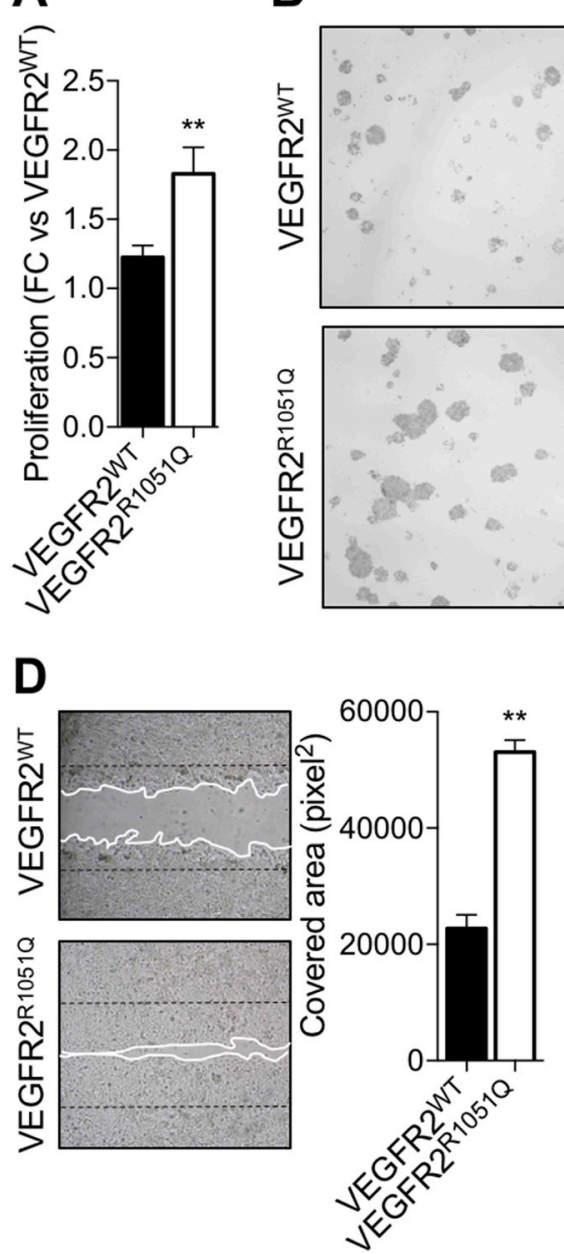

B

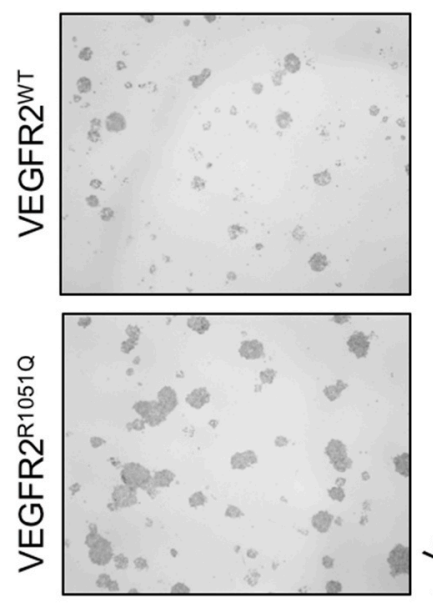

$\mathbf{E}$

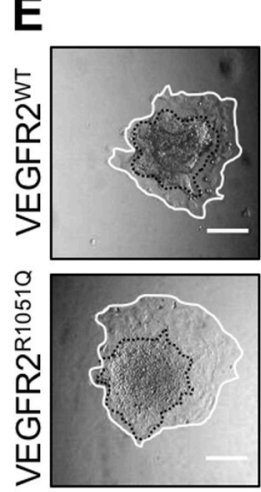

C

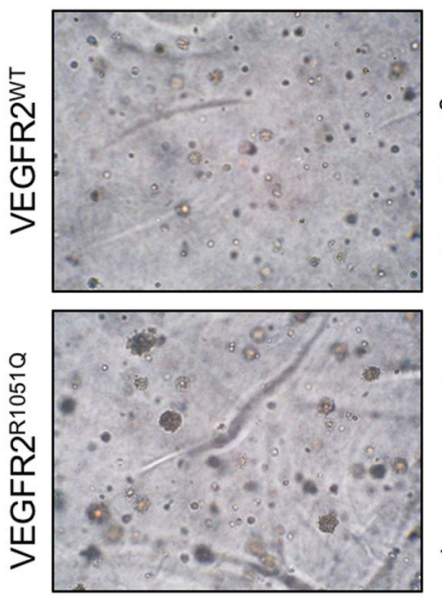

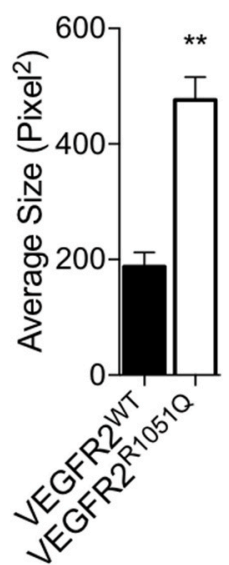
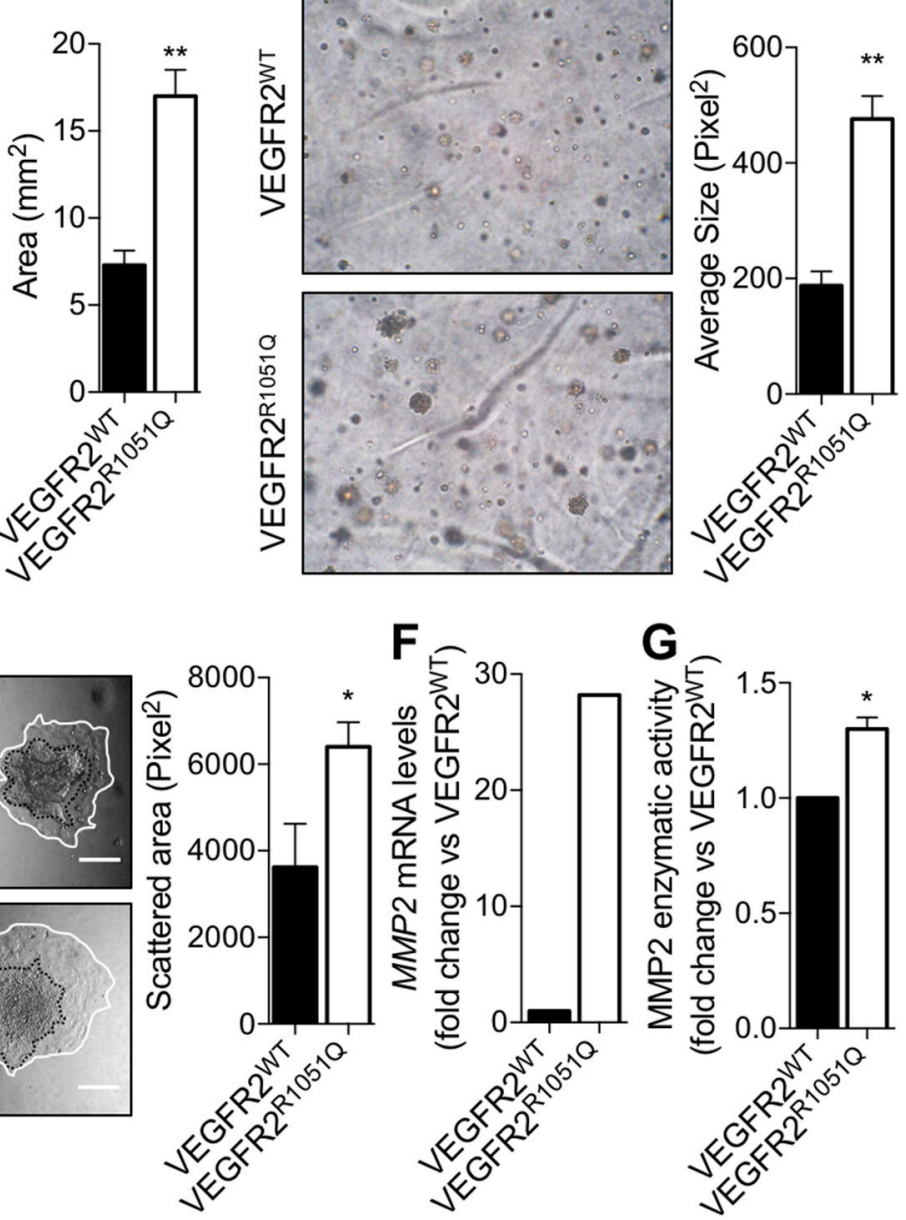

Fig. 3. In vitro analyses of Sk-Mel-31 tumor cells expressing VEGFR2 ${ }^{\text {R1051Q }}$. A, proliferation assay shown as fold change vs Sk-Mel-31-VEGFR2 ${ }^{\text {WT }}$. B-C, anchorage-dependent (B) and anchorage-independent (C) colony formation assays. Representative images and quantification of cell growth are shown. D, scratch assay. Representative pictures and quantification are shown. Scratch areas before and after 16-h incubation are highlighted (black dashed line and solid white lines, respectively). E, invasion of fibrin gel-embedded 3D spheroids. Representative images are shown and invaded area (highlighted in white) was quantified by ImageJ analysis software. Scale bar: $500 \mu \mathrm{m}$. F, mRNA levels of matrix metalloproteinase 2 (MMP2) analyzed by quantitative PCR. G, MMP2 activity measured in the conditioned medium of Sk-Mel-31-VEGFR2 ${ }^{\text {WT }}$ and Sk-Mel-31-VEGFR2 ${ }^{\text {R1051Q }}$ cells by gel zymography. Data are shown as mean \pm SEM. *, p $<0.05,{ }^{* *}, \mathrm{p}<0.01$, Student's $t$-test versus Sk-Mel-31-VEGFR2 ${ }^{\mathrm{WT}}$.

which are recurrently and similarly mutated. Here we focused on the missense mutations at positions 255 and 256 of the consensus sequence of the PK_Tyr_Ser-Thr protein domain. These mutations lay in the activation loop of the TKD and correspond to various well-known mutations of various members of the protein family. For instance, mutation of T599 and V600 residues in B-Raf, equivalent to positions 255 and 256 of the consensus sequence, increase the activity and downstream signaling of this oncogene $[18,28]$ and alter the response to targeted drugs. Furthermore, the amino acid substitution of D835 in FLT3 (corresponding to consensus position 256) is the most frequent genetic alteration in acute myeloid leukemia. This mutation constitutively activates FLT3, conferring a poorer prognosis [29,30]. In addition, mutation of residue 256 in EGFR, corresponding to L861, is an uncommon mutation associated with an altered response to the TKis gefitinib and erlotinib [31]. The same mutation in c-KIT, corresponding to residue D816, is associated with adverse prognosis in systemic mastocytosis and in acute myeloid leukemia [32]. In keeping with this data, VEGFR2 $2^{\mathrm{R} 1051 \mathrm{Q}}$ and VEGFR2 $2^{\text {D1052N }}$ are endowed with increased enzymatic activity and reduced membrane lateral diffusion. RTK braking is typically due to ligand-induced receptor localization in specific membrane micro-domains or interaction with other cellular components [33,34].
The stimulus-independent reduction of the membrane diffusion of VEGFR2 mutants and their stimulus-independent kinase activity support the hypothesis that these variants are in a constitutively active state. Accordingly, expression of VEGFR2 mutants results in increased activation of VEGFR2 downstream signaling (i.e. total phospho-tyrosine levels), augmented proliferation, tumorigenesis and metastasis. Remarkably, a co-occurrence analysis revealed that VEGFR2 mutations R1051Q and D1052N are mutually exclusive with B-Raf mutations. This suggests that VEGFR2 mutations may act in pathways regulating cell proliferation that are alternative versus those activated by B-Raf mutations.

A more in-depth characterization of the VEGFR2 ${ }^{\mathrm{R} 1051 \mathrm{Q}}$ mutant showed that it triggers the activation of a pro-oncogenic signaling program when expressed in tumor cells, that eventually results in increased tumor growth and metastasis. In addition, the expression of the VEGFR2 ${ }^{\text {R1051Q }}$ mutant stimulates ECM remodeling with increased metalloprotease activity, collagen production, leading to altered tissue architecture of tumor xenografts and promoting a more pronounced metastatic capacity. Together, these data support the hypothesis that the expression of VEGFR2 $2^{\mathrm{R} 1051 \mathrm{Q}}$ triggers the activation of classical RTK downstream signaling in cancer cells leading to reshaping of the tumor 
A

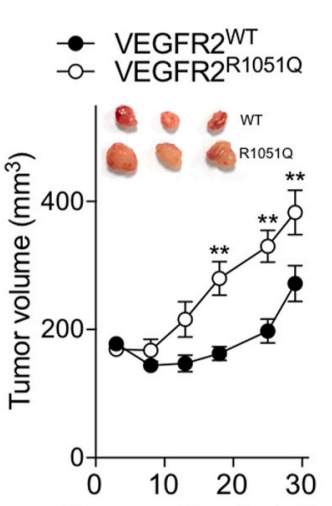

Days post-implantation
B
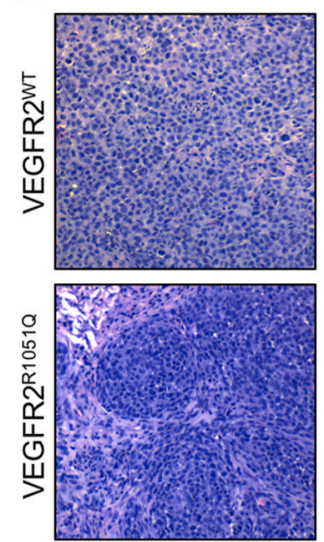

pVEGFR2 DAPI
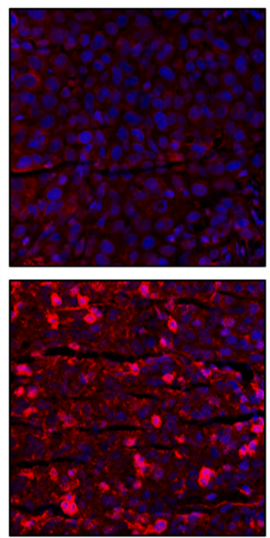

CD31 aSMA DAPI
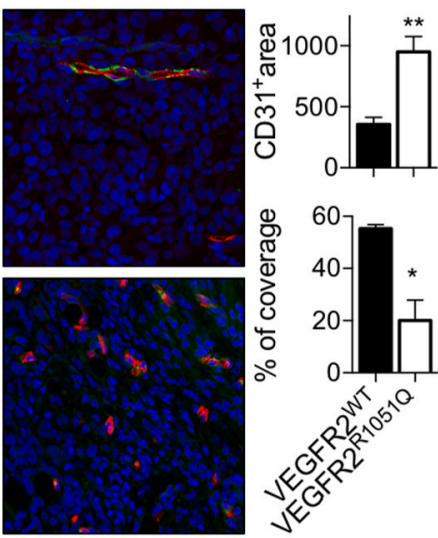
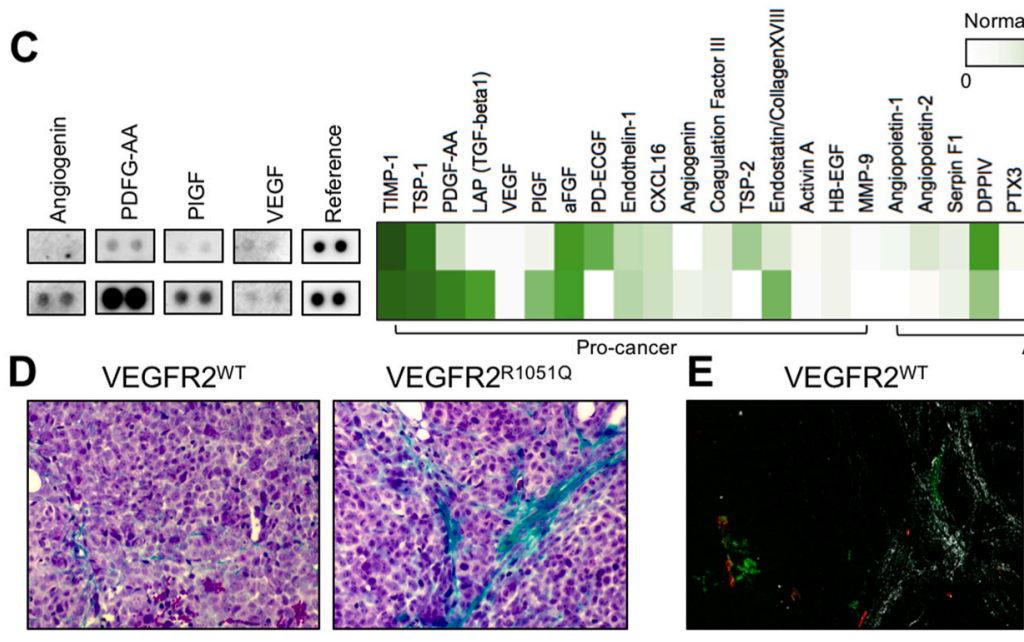

Normalized protein levels (RU)
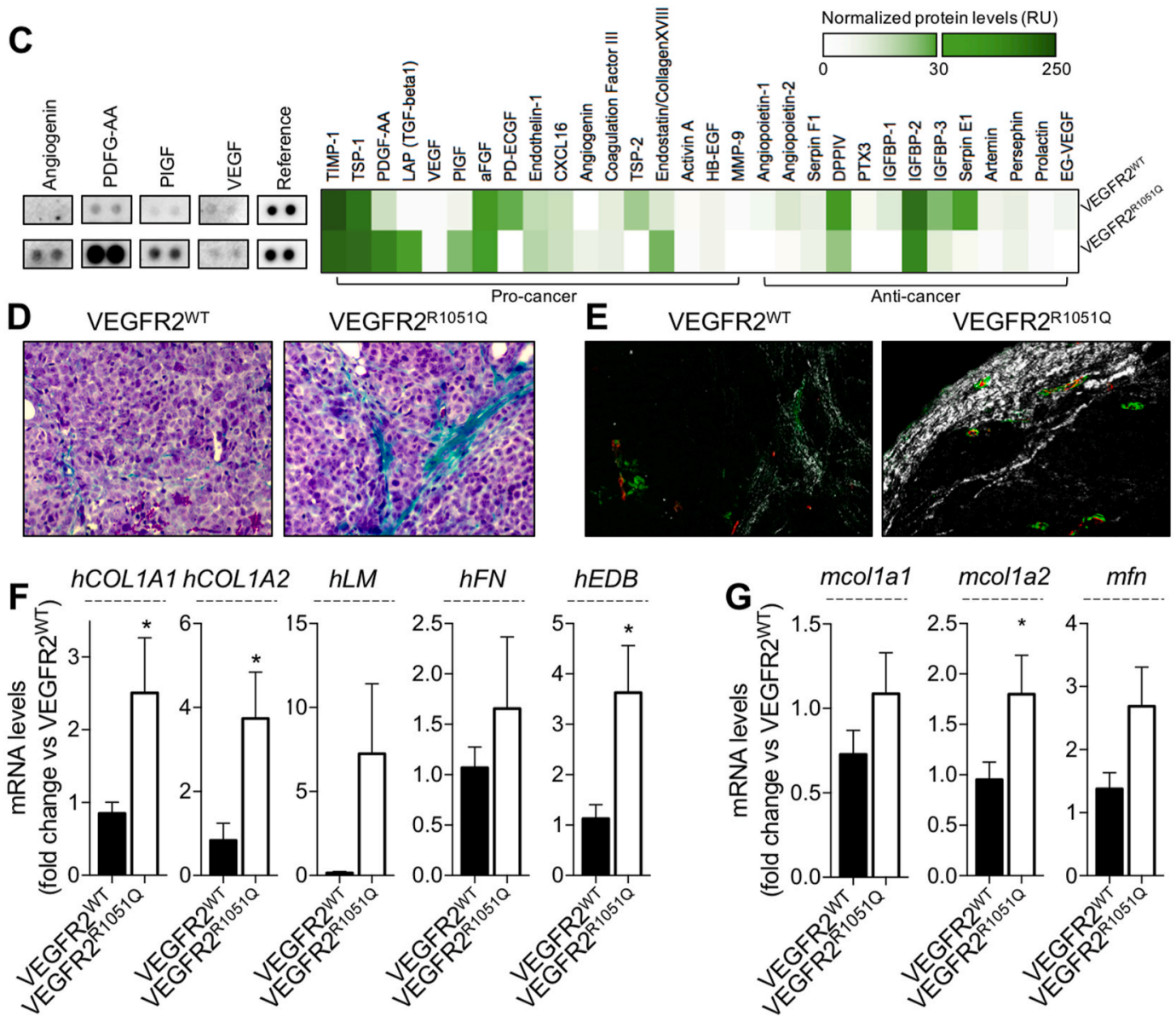

Fig. 4. In vivo analysis of the tumorigenic capacity of Sk-Mel-31 melanoma cells expressing VEGFR2 ${ }^{\text {R1051Q }}$. A, Cells were injected subcutaneously into the flank of 8-week-old NOD/SCID mice ( $\mathrm{n}=6-8 \mathrm{mice} /$ group) and tumor volume was monitored over time. Representative pictures of harvested tumors are shown in the inset. B, formalin-fixed paraffin-embedded tumor sections were stained with hematoxylin and eosin (H\&E) or immunodecorated for p-VEGFR2, CD31 or $\alpha$-SMA. Cell nuclei were counterstained with DAPI. Image quantification of $\mathrm{CD}^{+} 1^{+}$area and of vessel coverage with $\alpha$-SMA ${ }^{+}$mural cells were performed by ImageJ software. C, Human Angiogenesis Antibody Array on Sk-Mel-31-VEGFR2 ${ }^{\text {WT }}$ and Sk-Mel-31-VEGFR2 ${ }^{\text {R1051Q }}$-derived tumor total lysates. Spots corresponding to Angiogenin, PDGFAA, PIGF, VEGF and reference are shown. The heatmap shows the color-coded normalized protein levels of all expressed proteins (relative units - RU). D-E, Masson's trichrome (MT) staining (D) and second-harmonic generation (SHG) microscopy (E) of tumor sections. F-G, tumor mRNA levels of human (F) Collagen 1A1 ( $h$ COL1A1), Collagen 1A2 (hCOL1A2), Laminin $(h L M)$, Fibronectin $(h F N)$ and EDB fibronectin ( $h E D B)$ or murine (G) Collagen 1A1 (mcol1a1), Collagen 1A2 (mcol1a2) and Fibronectin $(m f n)$ analyzed by quantitative PCR. Data are shown as mean \pm SEM. ${ }^{*}, \mathrm{p}<0.05,{ }^{* *}, \mathrm{p}<0.01$, Student's $t$-test versus Sk-Mel-31-VEGFR2 ${ }^{\mathrm{WT}}$.

microenvironment, which in turn promotes tumor growth. Overall, our observations together with published data indicate that substitution of positions 255 and 256 alter the kinase activation mechanism making the receptor always active also in the absence of exogenous stimulation.

Various cancer-associated VEGFR2 mutations, other than R1051Q and D1052N substitutions, have been previously reported $[7,11,12]$.
Some of them are correlated to tumor growth and/or to altered response to targeted drugs, and include the substitutions D717V, G800D/R, L840F, G843D, S925F, R1022Q, L1049W, S1100F and R1032Q, the most frequent one. Among them L840F mutation induces therapy refractoriness in colon cancer patients $[7,8]$ while D717V, G800D/R, G843D, S925F, R1022Q, R1032Q and S1100F promote tumor growth in 


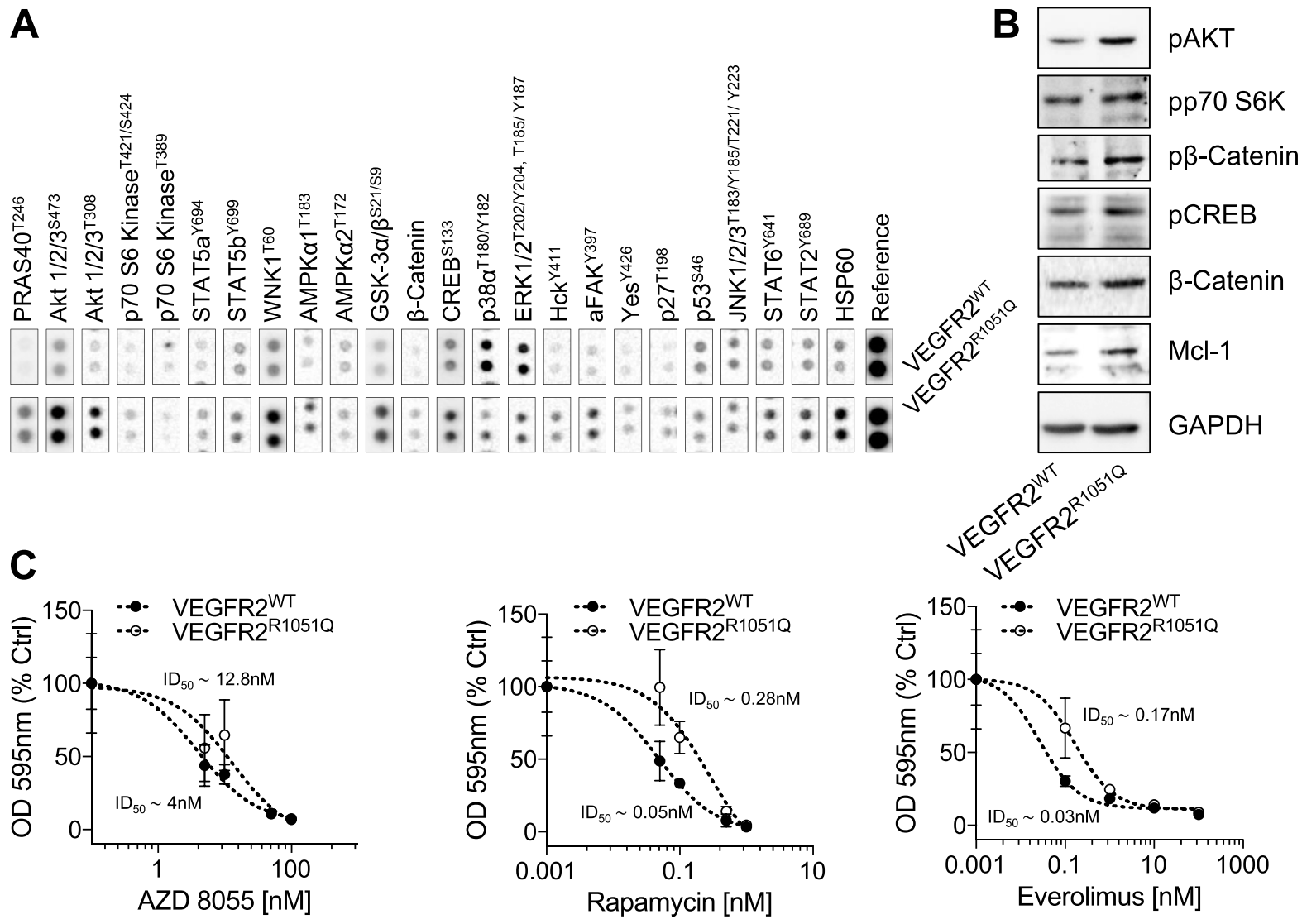

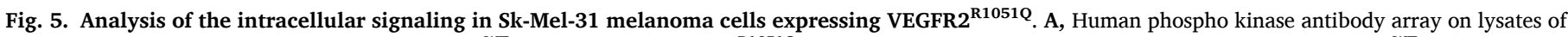

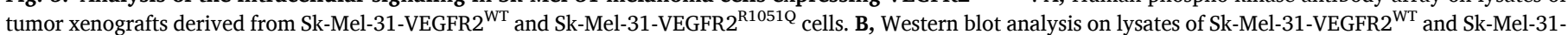

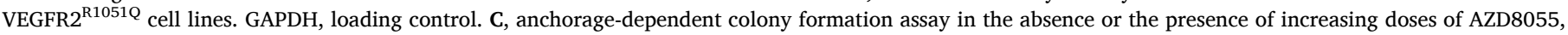
Rapamycin or Everolimus. Percentage $v s$ untreated cells was calculated and data are shown as mean \pm SEM. ID $_{50}$ values are shown for comparison.

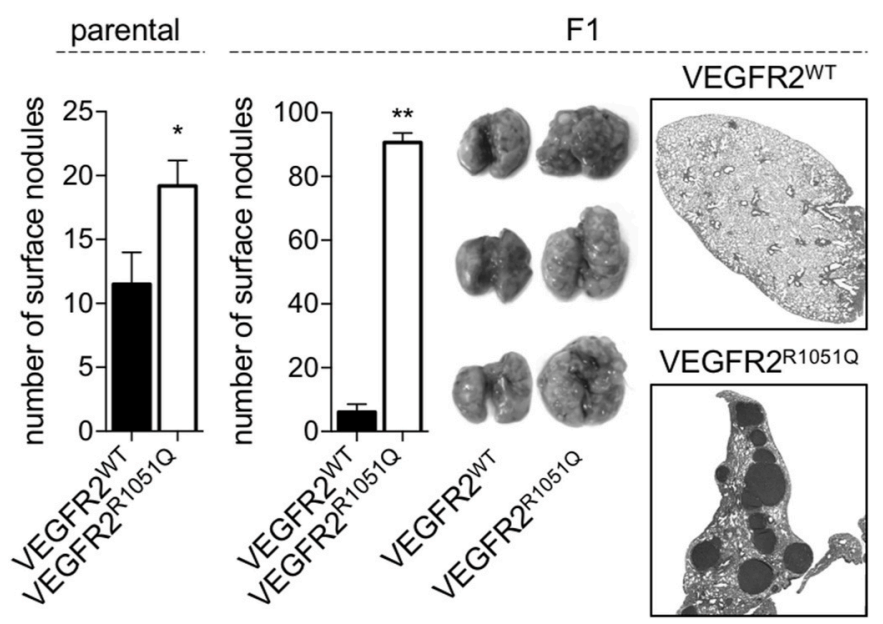

Fig. 6. Analysis of the metastatic capacity of Sk-Mel-31melanoma cells expressing VEGFR2 ${ }^{\text {R1051Q }}$. Parental or F1 generation melanoma cells were injected intravenously in NOD/SCID mice $(n=5)$. After 10 weeks, lungs were harvested and macroscopic surface nodules were counted. Representative pictures of harvested lungs and H\&E staining of formalin-fixed paraffin embedded lung sections are shown. Data are shown as mean \pm SEM. *, p $<0.05$, **, p $<$ 0.01 , Student's $t$-test versus Sk-Mel-31-VEGFR2 ${ }^{\mathrm{WT}}$.

a xenograft model of colon cancer [7,11,12]. A case report has shown that a patient affected by metastatic basal cell carcinoma expressing the substitution R1032Q of VEGFR2 had a good metabolic response to the treatment with pazopanib [12]. Here, LowMACA identified three previously uncharacterized VEGFR2 mutations (G922E, R1051Q and D1052N). Among them, R1051Q and D1052N substitutions were characterized as pro-oncogenic variants. Even though the mechanism by which other VEGFR2 mutations confer a pro-oncogenic role to this receptor remains partially unexplored [8], R1051Q and D1052N substitutions result in a ligand-independent activation of the receptor. Our data together with published ones provide strong evidence that somatic VEGFR2 variants, regardless rare, may have a deep impact on tumor progression.

TKi are widely used in clinics for the treatment of several cancer types [35]. In this frame, some TK mutations, including B-Raf ${ }^{\mathrm{V} 600}$, EGFR $^{\mathrm{L} 861}$ and VEGFR2 ${ }^{\mathrm{R} 961 / \mathrm{R} 1032}$ have been associated with a better response to TKi $[11,12,36,37]$. Similarly, here we show that the R1051Q mutation of VEGFR2 increases the sensitivity of cancer cells to the VEGFR2-targeted TKi Linifanib. Although further studies are warranted to confirm our findings, our results suggest that the screening with bioinformatics tools for TK protein mutations "analogous" to the R1051Q substitution in VEGFR2 might be predictive of prognosis and response to targeted therapy, with significant clinical implications.

\section{Authors' contributions}

Conceptualization: E.G., L.Z., S.M. Methodology: E.G., M.C., C.R., S. M. Formal analysis: E.G., S.M. Investigation: E.G., M.C., M.d.S., C.R. Writing original draft: E.G., S.M. Writing reviewing and editing: M.P., E. M. Project administration: S.M. Funding acquisition: M.P., S.M. 

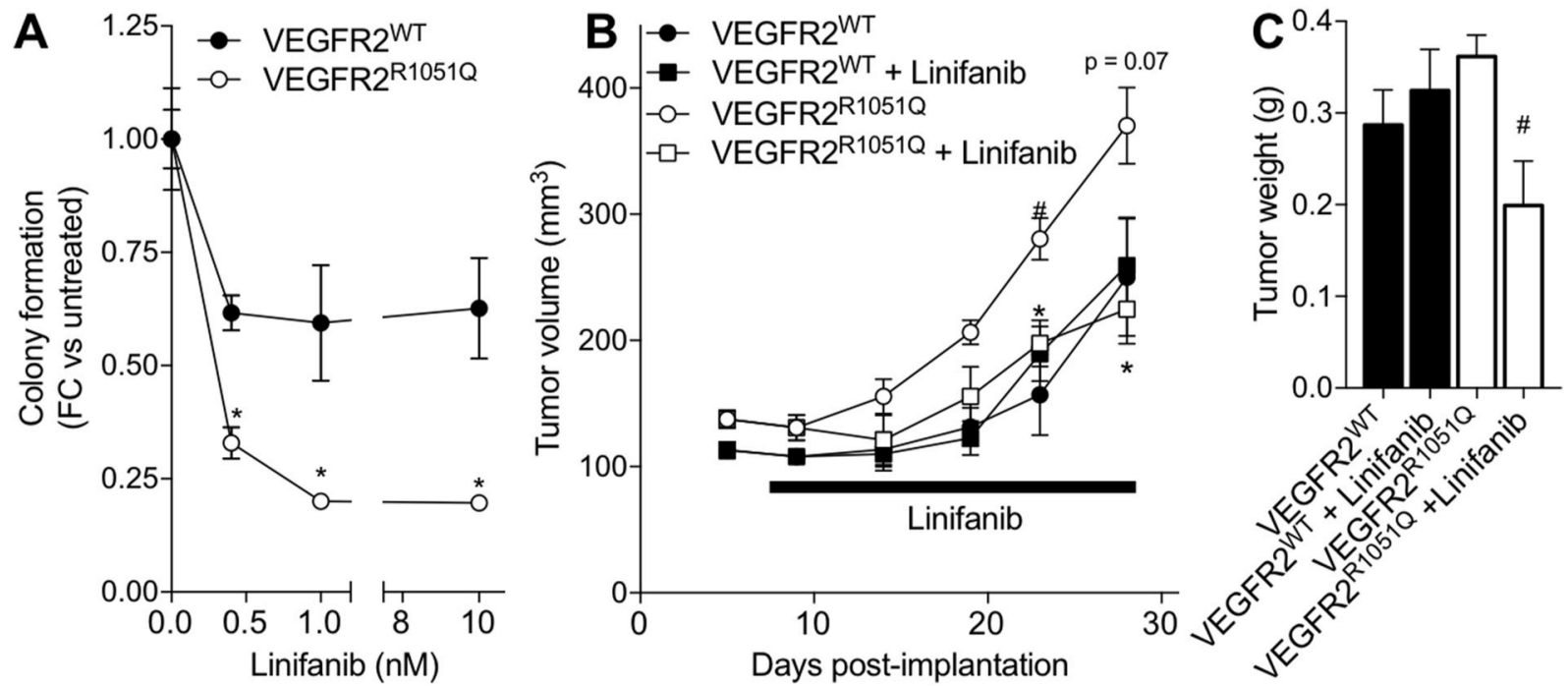

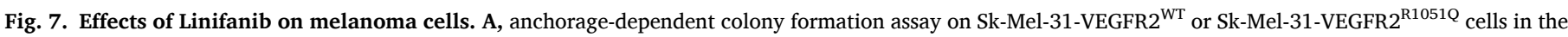

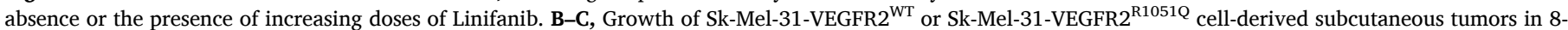

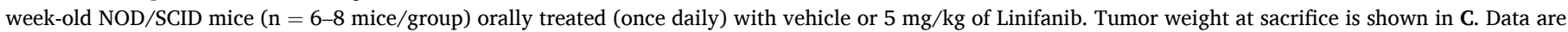
shown as mean \pm SEM. *, $\mathrm{p}<0.05$, Student's $t$-test versus Sk-Mel-31-VEGFR2 ${ }^{\mathrm{WT}}$. \#, $\mathrm{p}<0.05$, Student's $t$-test versus untreated Sk-Mel-31-VEGFR2 ${ }^{\mathrm{R} 1051 \mathrm{Q}}$.

\section{Acknowledgements}

This work was supported by grants from AIRC to S.M. (IG17276) and to M.P. (IG 2019 Id.23116). E.G. and M.d.S. were supported by FIRC Fellowships. E.G. was also supported by FUV Fellowship. MPP Lab was supported by Fondazione Cariplo and Regione Lombardia. The authors are grateful to Prof. Ballmer-Hofer for helpful discussion and for having provided plasmids, to Dr. Kalina Hristova for having provided plasmids, and to Dr. Nicolò Picchioni and Dr. Sara Femiano for technical help.

\section{Appendix A. Supplementary data}

Supplementary data to this article can be found online at https://doi. org/10.1016/j.canlet.2020.09.027.

\section{References}

[1] L. Holm, C. Sander, Mapping the protein universe, Science 273 (5275) (1996) 595-603.

[2] C. Chothia, et al., Evolution of the protein repertoire, Science 300 (5626) (2003) $1701-1703$.

[3] S. El-Gebali, et al., The Pfam protein families database in 2019, Nucleic Acids Res. 47 (D1) (2019) D427-D432.

[4] M.L. Miller, et al., Pan-cancer analysis of mutation hotspots in protein domains, Cell Syst 1 (3) (2015) 197-209.

[5] N.P. Gauthier, et al., MutationAligner: a resource of recurrent mutation hotspots in protein domains in cancer, Nucleic Acids Res. 44 (D1) (2016) D986-D991.

[6] G.E. Melloni, et al., LowMACA: exploiting protein family analysis for the identification of rare driver mutations in cancer, BMC Bioinf. 17 (2016) 80.

[7] R.A. Toledo, et al., Exome sequencing of plasma DNA portrays the mutation landscape of colorectal cancer and discovers mutated VEGFR2 receptors as modulators of antiangiogenic therapies, Clin. Canc. Res. 24 (15) (2018) 3550-3559.

[8] R.D. Kumar, R. Bose, Analysis of somatic mutations across the kinome reveals loss of-function mutations in multiple cancer types, Sci. Rep. 7 (1) (2017) 6418.

[9] Z. Du, C.M. Lovly, Mechanisms of receptor tyrosine kinase activation in cancer, Mol. Canc. 17 (1) (2018) 58.

[10] Y.B. Khotskaya, et al., Targeting TRK family proteins in cancer, Pharmacol. Ther. 173 (2017) 58-66.

[11] A. Loaiza-Bonilla, et al., KDR mutation as a novel predictive biomarker of exceptional response to regorafenib in metastatic colorectal cancer, Cureus 8 (2) (2016).

[12] T.C. Knepper, et al., Clinical response to pazopanib in a patient with KDR-mutated metastatic basal cell carcinoma, Jama Dermatology 153 (6) (2017) 607-609.

[13] S. Koch, L. Claesson-Welsh, Signal transduction by vascular endothelial growth factor receptors, Cold Spring Harb Perspect Med 2 (7) (2012), a006502.

[14] L. Lian, et al., VEGFR2 promotes tumorigenesis and metastasis in a pro-angiogenicindependent way in gastric cancer, BMC Canc. 19 (2019).
[15] S. Rezzola, et al., VEGFR2 activation mediates the pro-angiogenic activity of BMP4, Angiogenesis 22 (4) (2019) 521-533.

[16] V. Damioli, et al., Multi-physics interactions drive VEGFR2 relocation on endothelial cells, Sci. Rep. 7 (1) (2017) 16700.

[17] S. Mitola, et al., IL-12 regulates an endothelial cell-lymphocyte network: effect on metalloproteinase-9 production, J. Immunol. 171 (7) (2003) 3725-3733.

[18] M. Dankner, et al., Classifying BRAF alterations in cancer: new rational therapeutic strategies for actionable mutations, Oncogene 37 (24) (2018) 3183-3199.

[19] G.L. Picharski, et al., The impact of Flt3 gene mutations in acute promyelocytic leukemia: a meta-analysis, Cancers 11 (9) (2019).

[20] H. Kiyoi, T. Naoe, Biology, clinical relevance, and molecularly targeted therapy in acute leukemia with FLT3 mutation, Int. J. Hematol. 83 (4) (2006) 301-308.

[21] W.H. Hsu, et al., Overview of current systemic management of EGFR-mutant NSCLC, Ann. Oncol. 29 (suppl_1) (2018) i3-i9.

[22] T.H. Wu, et al., New data on clinical decisions in NSCLC patients with uncommon EGFR mutations, Expet Rev. Respir. Med. 11 (1) (2017) 51-55.

[23] A. Picca, et al., FGFR1 actionable mutations, molecular specificities, and outcome of adult midline gliomas, Neurology 90 (23) (2018) e2086-e2094.

[24] T. Iwata, et al., Highly activated Fgfr3 with the K644M mutation causes prolonged survival in severe dwarf mice, Hum. Mol. Genet. 10 (12) (2001) 1255-1264.

[25] A. Jacob, R. Prekeris, The regulation of MMP targeting to invadopodia during cancer metastasis, Front Cell Dev Biol 3 (2015) 4.

[26] S. Izraely, et al., The metastatic microenvironment: melanoma-microglia cross-talk promotes the malignant phenotype of melanoma cells, Int. J. Canc. 144 (4) (2019) $802-817$.

[27] K. Koras, et al., Feature selection strategies for drug sensitivity prediction, Sci. Rep. 10 (1) (2020) 9377.

[28] D. Schadendorf, et al., Melanoma. Lancet 392 (10151) (2018) 971-984.

[29] E. Bailey, et al., FLT3/D835Y mutation knock-in mice display less aggressive disease compared with FLT3/internal tandem duplication (ITD) mice, Proc. Natl. Acad. Sci. U. S. A. 110 (52) (2013) 21113-21118.

[30] R.J. Mattison, et al., Implications of FLT3 mutations in the therapy of acute myeloid leukemia, Rev. Recent Clin. Trials 2 (2) (2007) 135-141.

[31] J.Y. Wu, et al., Effectiveness of tyrosine kinase inhibitors on "uncommon epidermal growth factor receptor mutations of unknown clinical significance in non-small cell lung cancer, Clin. Canc. Res. 17 (11) (2011) 3812-3821.

[32] K.V. Gleixner, et al., KIT-D816V-independent oncogenic signaling in neoplastic cells in systemic mastocytosis: role of Lyn and Btk activation and disruption by dasatinib and bosutinib, Blood 118 (7) (2011) 1885-1898.

[33] J. Lippincott-Schwartz, E. Snapp, A. Kenworthy, Studying protein dynamics in living cells, Nat. Rev. Mol. Cell Biol. 2 (6) (2001) 444-456.

[34] R. Amodeo, et al., Molecular insight on the altered membrane trafficking of TrkA kinase dead mutants, Biochim. Biophys. Acta Mol. Cell Res. (2) (2020) 1867.

[35] Q. Jiao, et al., Advances in studies of tyrosine kinase inhibitors and their acquired resistance, Mol. Canc. 17 (1) (2018) 36.

[36] T.J. Lynch, et al., Activating mutations in the epidermal growth factor receptor underlying responsiveness of non-small-cell lung cancer to gefitinib, N. Engl. J. Med. 350 (21) (2004) 2129-2139.

[37] E.W. Joseph, et al., The RAF inhibitor PLX4032 inhibits ERK signaling and tumor cell proliferation in a V600E BRAF-selective manner, Proc. Natl. Acad. Sci. U. S. A. 107 (33) (2010) 14903-14908. 